\title{
Modifying the Structure and Dynamics of Ionomers Through Counterion Sterics
}

Joshua S. Enokida ${ }^{1}$, Weiguo Hu${ }^{1}$, Huagao Fang ${ }^{2}$, Brian F. Morgan ${ }^{3}$, Frederick L. Beyer ${ }^{3}$, H. Henning Winter ${ }^{2}$, and E. Bryan Coughlin ${ }^{1 *}$

${ }^{1}$ Department of Polymer Science and Engineering, University of Massachusetts Amherst

${ }^{2}$ Department of Chemical Engineering, University of Massachusetts Amherst

${ }^{3}$ U.S. Army Research Laboratory, Aberdeen Proving Ground, MD

\section{Supporting Information}

\section{Determination of Copolymer Composition}

The composition of each copolymer was determined by comparing the integrations of the aromatic DMOASS peaks with the vinylic isoprene peaks in the ${ }^{1} \mathrm{H}$ NMR spectrum as shown in Figure $\mathrm{S} 1$. The aromatic peaks were used as the reference and each integration was normalized by the corresponding number of protons. Three isomers are formed during the polymerization of isoprene: 1,4-addition, 1,2-addition, and 3,4- addition. The vinylic protons associated with each isomer possess distinct chemical shifts between $\delta 6-4.5 \mathrm{ppm}$, thus the relative ratios of each isomer can be estimated. The quantity of 1,2- and 1,4-additions were determined directly from their corresponding integrations. The 3,4-addition was calculated by integrating over the entire 1,2- and 3,4-addition overlapping region ( $\delta 5.0-4.50 \mathrm{ppm})$. The two protons corresponding to the 1,2-addition were subtracted out and the remainder was normalized by the two protons to give the quantity of 3,4-addition. For the P(I-ran-SS)-7.2-DMOA copolymers series, the isoprene contained relative ratios of 87.8 to 4.2 to 8.1 of the $1,4-, 1,2-$, and $3,4-$ isomers, respectively. 


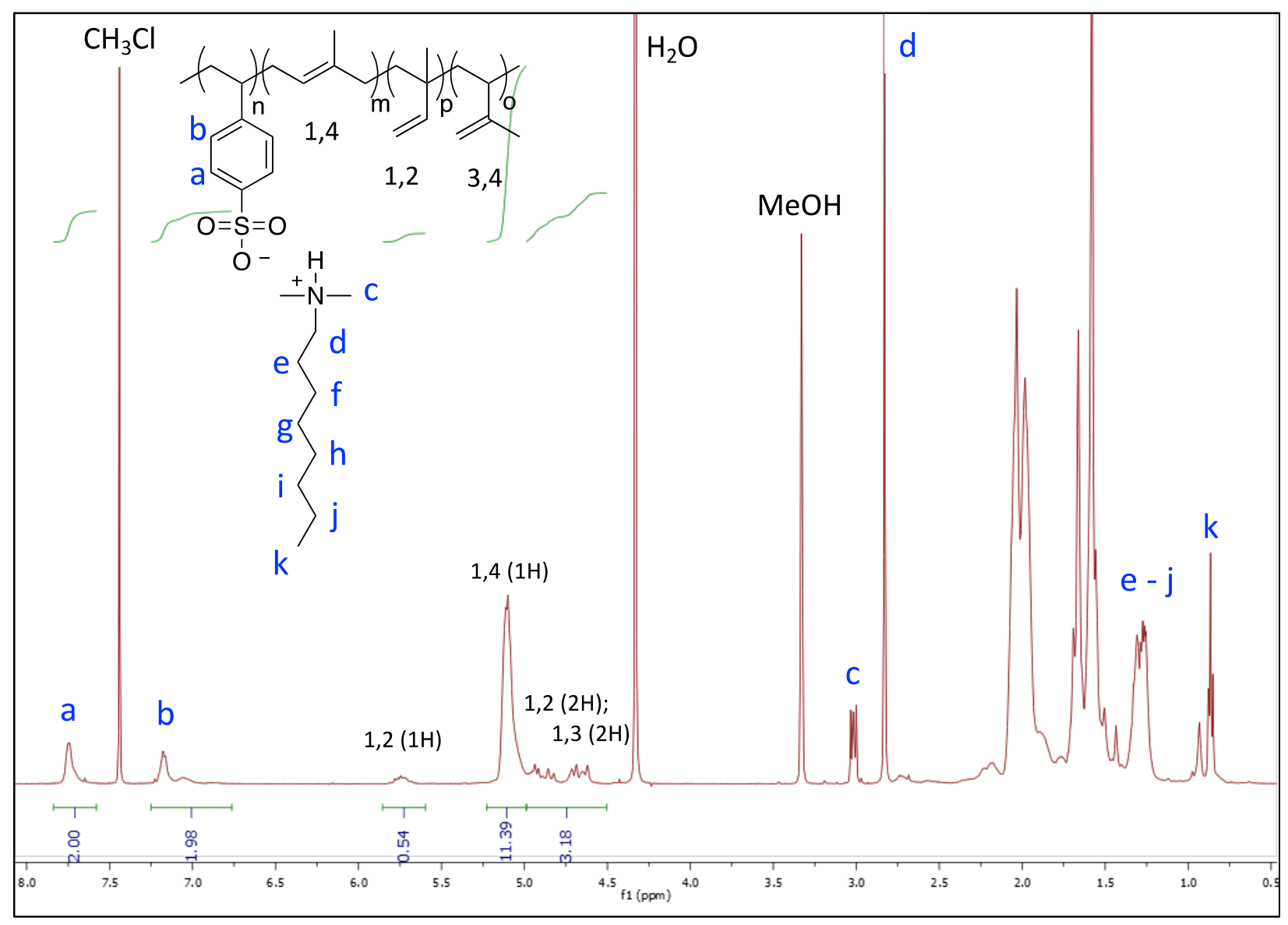

Figure S1. ${ }^{1} \mathrm{H}$ NMR spectrum for a P(I-ran-SS)-7.2-DMOA copolymer.

${ }^{1} \mathrm{H}$ NMR (500 MHz, Chloroform-d1:Methanol-d4, 2:1, v/v): $\delta 7.75$ (2H, aromatic), $7.18(2 \mathrm{H}$, aromatic), $5.74\left(1 \mathrm{H}, \mathrm{CH}_{2}=\mathrm{CH}-\right), 5.10\left(1 \mathrm{H},-\mathrm{CH}_{2}-\mathrm{CH}=\mathrm{C}-\right), 5.00-4.50\left(2 \mathrm{H}, \mathrm{CH}_{2}=\mathrm{CH}-; 2 \mathrm{H}, \mathrm{CH}_{2}=\mathrm{C}-\right.$ $\left.\mathrm{CH}_{3}\right), 3.02\left(2 \mathrm{H}, \mathrm{N}-\mathrm{CH}_{2}-\right), 2.83\left(6 \mathrm{H}, \mathrm{N}-\mathrm{CH}_{3}\right), 1.37-1.20\left(12 \mathrm{H}, \mathrm{CH}_{2}-\mathrm{CH}_{2}-\mathrm{CH}_{2}\right), 0.87\left(3 \mathrm{H}, \mathrm{CH}_{2}-\right.$ $\mathrm{CH}_{3}$ ), 2.30-0.80 (Backbone, $-\mathrm{CH}_{2-},-\mathrm{CH}_{3}$ ). 


\section{Degree of Polymerization Calculation}

The degree of polymerization (DP) was calculated as follows. ${ }^{1} \mathrm{H}$ NMR of the crude product was used to determine the DMOASS conversion (X DMOASS). The moles of reacted DMOASS ( $\mathrm{N}_{\mathrm{r}, \mathrm{DMOASS}}$ ) was determined by multiplying the initial DMOASS input $\left(\mathrm{N}_{\mathrm{i}, \mathrm{DMOASS}}\right)$ by its conversion.

$$
N_{r, D M O A S S}=N_{i, D M O A S S} \times X_{D M O A S S}
$$

Then, $\mathrm{N}_{\mathrm{r}, \mathrm{DMOASS}}$ was multiplied by the mole ratio of the final copolymer composition ( $\left.\mathrm{r}_{\mathrm{Iso}}\right)$ to give the moles of reacted $\mathrm{I}\left(\mathrm{N}_{\mathrm{r}, \mathrm{Iso}}\right)$.

$$
N_{r, I S O}=N_{r, D M O A S S} \times r_{I S O}
$$

By summing $\mathrm{N}_{\mathrm{r}, \mathrm{DMOASS}}$ and $\mathrm{N}_{\mathrm{r}, \mathrm{I}}$ and dividing by the moles of initator $\left(\mathrm{N}_{\mathrm{i}, \text { In }}\right)$ an estimation of the DP can be determined.

$$
D P=\frac{N_{r, D M O A S S}+N_{r, I s o}}{N_{i, I n}}
$$

Calculation for P(I-ran-SS)-7.2-DMOA is shown below.

$$
\begin{gathered}
N_{r, \text { DMOASS }}=25 \mathrm{mmol} \times 0.38=9.5 \mathrm{mmol} \\
N_{r, I S o}=9.5 \mathrm{mmol} \times\left(\frac{92.8}{7.2}\right)=122.4 \mathrm{mmol} \\
D P=\frac{9.5 \mathrm{mmol}+122.4 \mathrm{mmol}}{0.1036 \mathrm{mmol}}=1270
\end{gathered}
$$




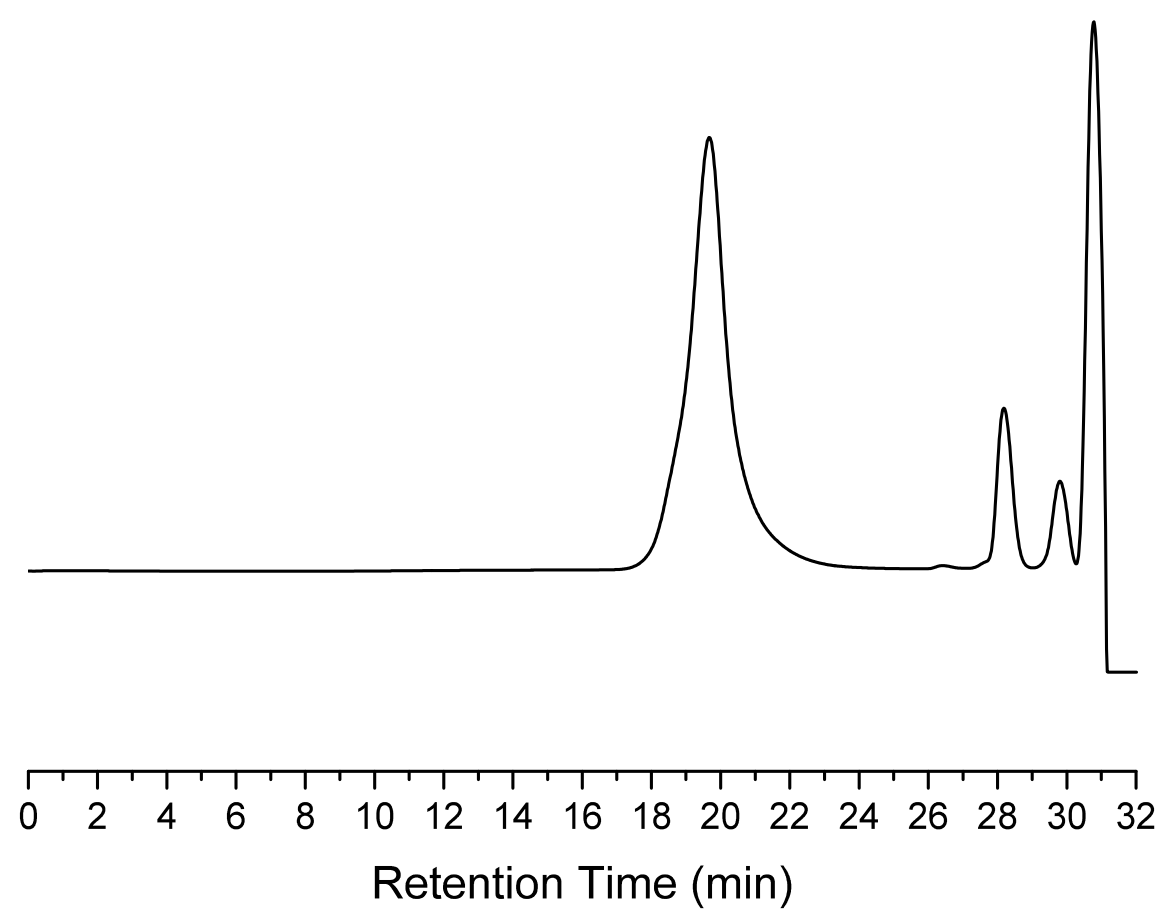

Figure S2. Gel permeation chromatography trace of polyisoprene homopolymer control.

The relative molecular weights and dispersity of the polyisoprene homopolymer synthesized under the same reaction conditions as the P(I-ran-SS)-7.2-DMOA copolymer was determined using a gel permeation chromatography with THF as the eluent and PS calibration curve. The $M_{n}, M_{w}$, and $Đ$ for the polyisoprene homopolymer were $63,800 \mathrm{~g} / \mathrm{mol}, 82700 \mathrm{~g} / \mathrm{mol}$, and 1.3 , respectively.

\section{${ }^{1} \mathrm{H}$ NMR Spectroscopy of P(I-ran-SS)-7.2-x Copolymers}

Figure S2 shows ${ }^{1} \mathrm{H}$ NMR spectra of the counterion exchanged copolymers. The protons on the carbon alpha to the nitrogen appeared between $\delta 2.5-3.5 \mathrm{ppm}$ for all of the counterions. Signals appearing at $\delta 3.00$ and $\delta 2.80 \mathrm{ppm}$ for the DMOA counterion correspond to the 2 methylene protons and the 6 methyl protons, respectively. After counterion exchange, these signals 
disappeared and were replaced by one signal between $\delta 3.10$ and $\delta 3.30 \mathrm{ppm}$ corresponding to 12 methyl protons for the TMA-containing copolymer or 8 methylene protons for the TEA, TPA, and TBA-containing copolymers. No peak appear in this range for the sodium-containing copolymer.

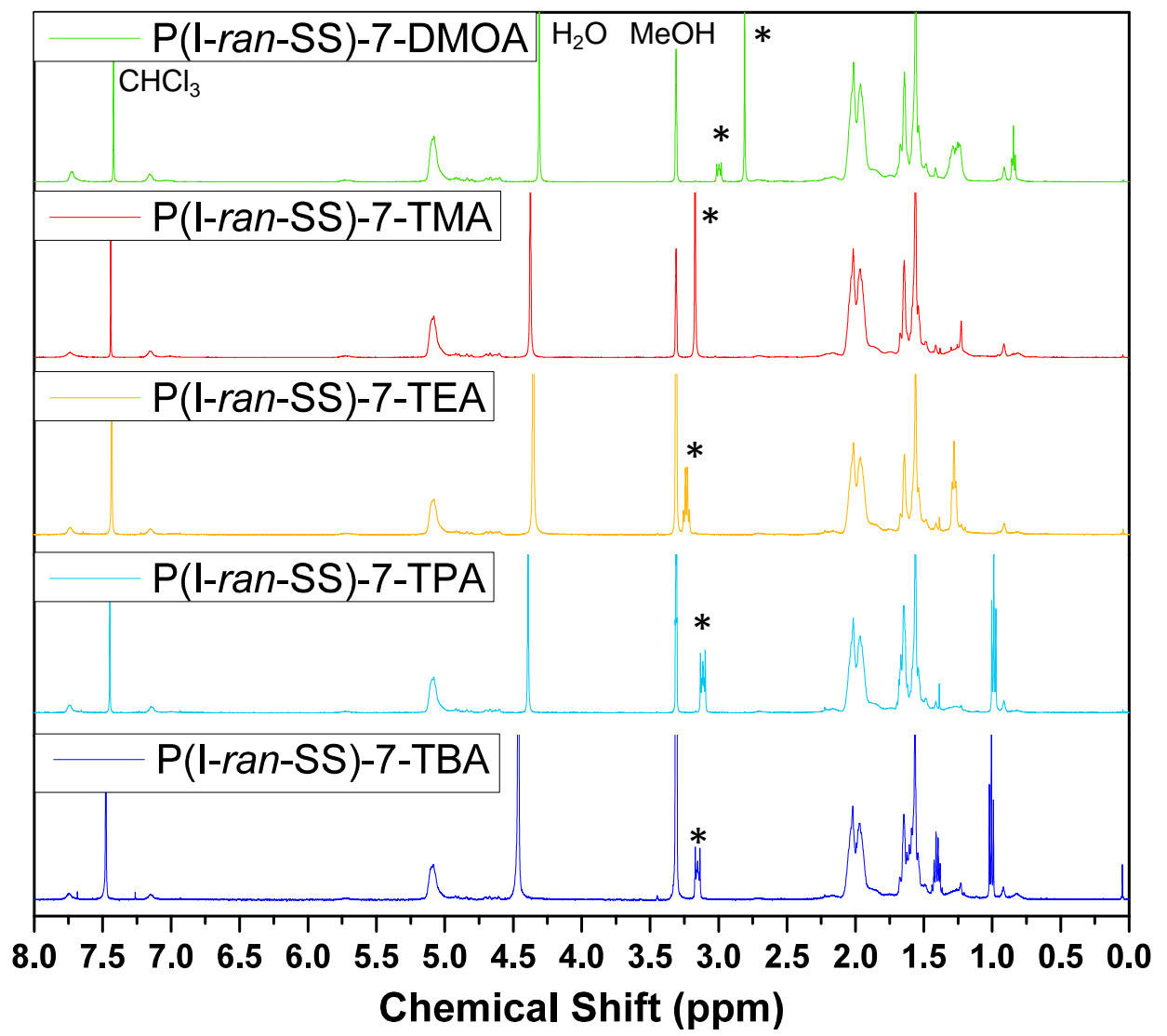

Figure S3. Comparison of the ${ }^{1} \mathrm{H}$ NMR spectrum for the P(I-ran-SS)-7.2-x copolymer series (x $=$ DMOA, TMA, TEA, TPA, and TBA). The asterisk marks the counterion methyl(ene) protons adjacent to the nitrogen. 


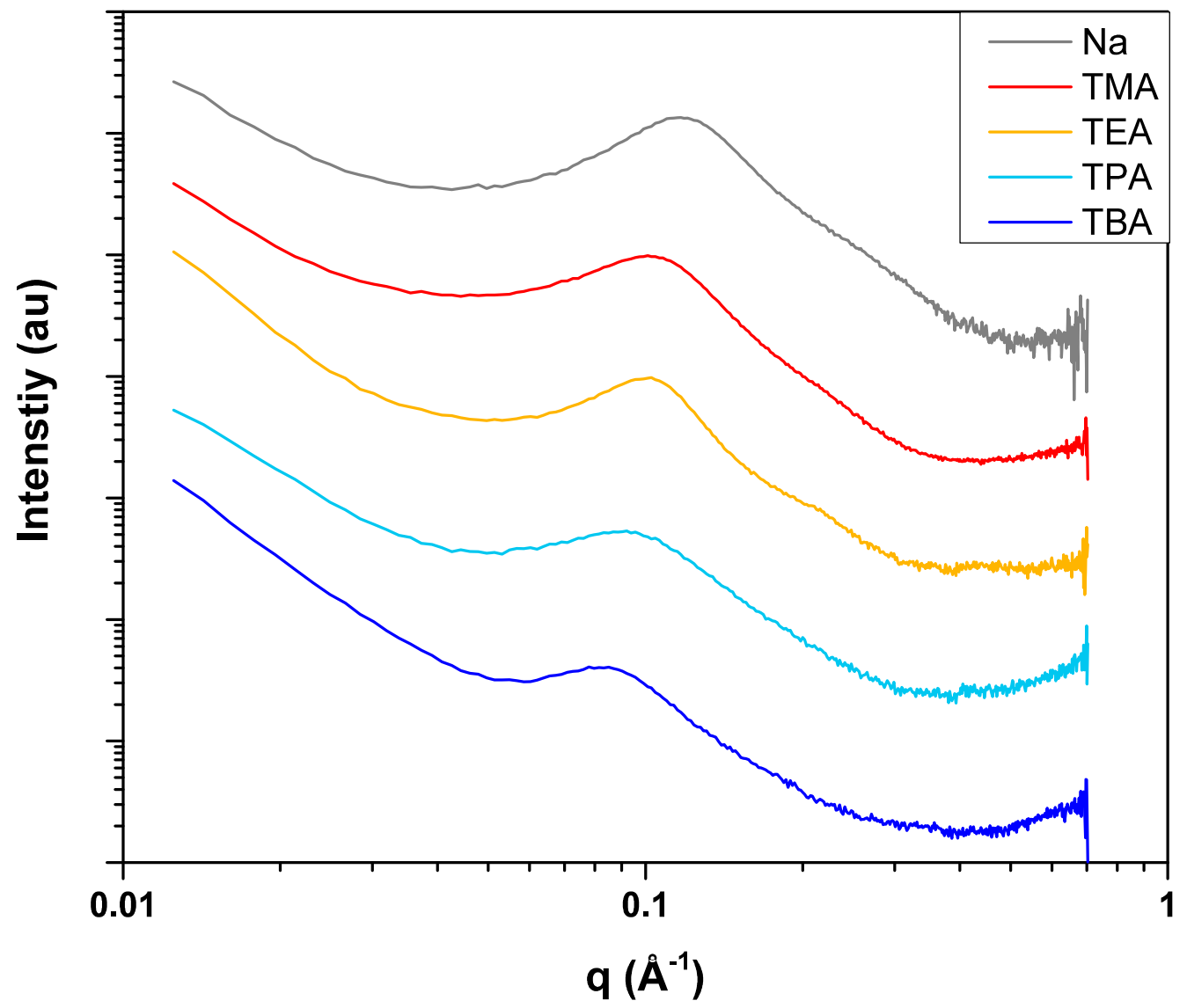

Figure S4. Offset SAXS profiles for the P(I-ran-SS)-7.2-x copolymers with different counterions $\left(\mathrm{x}=\mathrm{Na}^{+}\right.$, TMA, TEA, TPA, and TBA $)$. 
The scattering intensity $(I(q))$ is defined by the hard-sphere form factor $(\Phi(x))$ and a PercusYevick function that accounts for inter-cluster scattering interference as shown below. ${ }^{1}$

$$
I(q)=A \Phi^{2}(x)\left(\frac{1}{1+24 \eta\left(\frac{G(B)}{B}\right)}\right)
$$

where

$$
\begin{gathered}
\Phi(x)=3 \frac{\sin (x)-x \cos (x)}{x^{3}} \\
x=q R_{1} \\
\eta=\frac{4}{3} \pi R_{c a}^{3}\left(\frac{1}{V_{p}}\right) \\
B=2 q R_{c a} \\
G(B)=\frac{(1+2 \eta)^{2}}{B^{2}(1-\eta)^{4}}(\sin B-B \cos B)-\frac{6 \eta\left(1+\frac{\eta}{2}\right)^{2}}{B^{3}(1-\eta)^{4}}\left[2 B \sin B+\left(2-B^{2}\right) \cos B-2\right]+ \\
\frac{\eta(1+2 \eta)^{2}}{2 B^{5}(1-\eta)^{4}}\left\{-B^{4} \cos B+4\left[\left(3 B^{2}-6\right) \cos B+\left(B^{3}-6 B\right) \sin B+6\right]\right\}
\end{gathered}
$$

\section{Ion Aggregate Composition Calculations}

Table $\mathrm{S} 1$ shows the volumes of the tetraalkylammonium chloride molecules $\left(V_{\text {cation-Cl }}\right)$ and the adjusted volumes $\left(V_{\text {cation- }-\mathrm{SO}_{3}}\right)$ used for the ion aggregate composition calculations. These estimated adjusted volumes account for the volumetric difference between a chloride and sulfonate anion $\left(\mathrm{V}_{\mathrm{SO}_{3}-\mathrm{Cl}}\right)$ which was determined using the equation shown below.

$$
V_{\mathrm{SO}_{3}-\mathrm{Cl}}=\frac{5}{7} \mathrm{Na}_{2} \mathrm{SO}_{4}-V_{\mathrm{NaCl}}
$$


The volume of the sodium sulfate was multiplied by a factor of $5 / 7$ to adjust for the additional sodium cation and oxygen. ${ }^{2,3}$ The difference in volume was found to be $0.018 \mathrm{~nm}^{3}$ which was added to the $V_{\text {cation-Cl }}$ for each tetraalkylammonium.

Table S1. Volumes of the Tetraalkylammonium Chloride and Adjusted Values for Tetraalkylammonium Sulfonate Estimations

\begin{tabular}{ccccc}
\hline Sample & $\begin{array}{c}\mathbf{M W} \\
(\mathbf{g} / \mathbf{m o l})\end{array}$ & $\begin{array}{c}\rho \\
\left(\mathbf{g} / \mathbf{c m}^{3}\right)\end{array}$ & $\begin{array}{c}\boldsymbol{V}_{\text {cation-Cl }} \\
\left(\mathbf{n m}^{3}\right)\end{array}$ & $\begin{array}{c}\boldsymbol{V}_{\text {cation-SO }} \\
\left(\mathbf{n m}^{3}\right)\end{array}$ \\
\hline $\mathrm{Na}_{2} \mathrm{SO} 4$ & 142.04 & 2.664 & 0.089 & \\
$\mathrm{NaCl}$ & 58.44 & 2.16 & 0.045 & \\
$\mathrm{TMACl}$ & 109.06 & 1.17 & 0.155 & 0.173 \\
$\mathrm{TEACl}$ & 165.71 & 1.08 & 0.255 & 0.273 \\
$\mathrm{TPACl}$ & 221.81 & 1.033 & 0.357 & 0.375 \\
$\mathrm{TBACl}$ & 277.92 & 1.05 & 0.440 & 0.458 \\
\hline
\end{tabular}

Additionally, Table $\mathrm{S} 2$ shows values used in the calculation of $\mathrm{N}_{\mathrm{agg}}\left(\mathrm{V}_{\mathrm{p}}\right)$. The volume fraction of the styrenesulfonate-cation ( $\left.\phi_{\mathrm{ss}}\right)$ for each tetraalkylammonium was estimated using the mole fraction of isoprene and styrenesulfonate in the copolymer ( 0.93 and 0.07 , respectively) in addition to their corresponding densities $\left(\rho P I=0.91 \mathrm{~g} / \mathrm{cm}^{3}, \rho P S S S=1.11 \mathrm{~g} / \mathrm{cm}^{3}\right)$. Since the density of the styrenesulfonate-tetraalkylammoniums is not known, the density of poly(sodium styrenesulfonate) was used as an approximation, assuming that the density does not significantly change with the different counterions. Thus, the calculated volume fractions scale with their molecular weights. Furthermore, the number of styrenesulfonate-cation pairs per volume ( $\left.\eta_{\mathrm{SS}}\right)$ was calculated using similar assumptions and is shown below, 


$$
\eta_{S S}=6.022 \times 10^{23} \frac{\rho_{S S}}{M W_{S S-c a t i o n}}
$$

where the $\rho s s$ is expressed in units of $\mathrm{g} / \mathrm{nm}^{3}$.

Table S2. Tetraalkylammonium Styrenesulfonate Volume Fraction ( $\phi$ ss), Number of Ion Pairs per Volume $(\eta s s)$, and Volume of Sample per Aggregate $\left(V_{p}\right)$ Values Used for the $N_{\text {agg }}\left(V_{p}\right)$ Calculation

\begin{tabular}{ccccc}
\hline Sample & $\phi \mathbf{s s}$ & $\eta_{\mathbf{s s}}$ & $\mathbf{V}_{\mathbf{p}}$ & $\mathbf{N}_{\mathbf{a g g}}\left(\mathbf{V}_{\mathbf{p}}\right)$ \\
\hline PSSS & 0.157 & 3.24 & 234 & 119 \\
PTMASS & 0.189 & 2.60 & 383 & 188 \\
PTEASS & 0.221 & 2.13 & 385 & 182 \\
PTPASS & 0.251 & 1.81 & 656 & 298 \\
PTBASS & 0.278 & 1.57 & 902 & 394 \\
\hline
\end{tabular}

$$
N_{a g g}\left(V_{p}\right)=\phi_{S S} V_{p} \eta_{S S}
$$

Table S3. Summary of Ion Aggregate Composition

\begin{tabular}{cccc}
\hline Sample & $\mathbf{N}_{\text {agg }}\left(\mathbf{R}_{1}\right)$ & $\mathbf{N}_{\text {agg }}\left(\mathbf{V}_{\mathbf{p}}\right)$ & $\mathbf{N}_{\text {agg }}\left(\mathbf{V}_{\mathbf{p}}\right) / \mathbf{N}_{\text {agg }}\left(\mathbf{R}_{\mathbf{1}}\right)$ \\
\hline Na & 220 & 120 & 0.5 \\
TMA & 140 & 190 & 1.3 \\
TEA & 120 & 180 & 1.5 \\
TPA & 65 & 300 & 4.6 \\
TBA & 73 & 390 & 5.4 \\
\hline
\end{tabular}




\section{Variable Temperature SAXS Profiles}

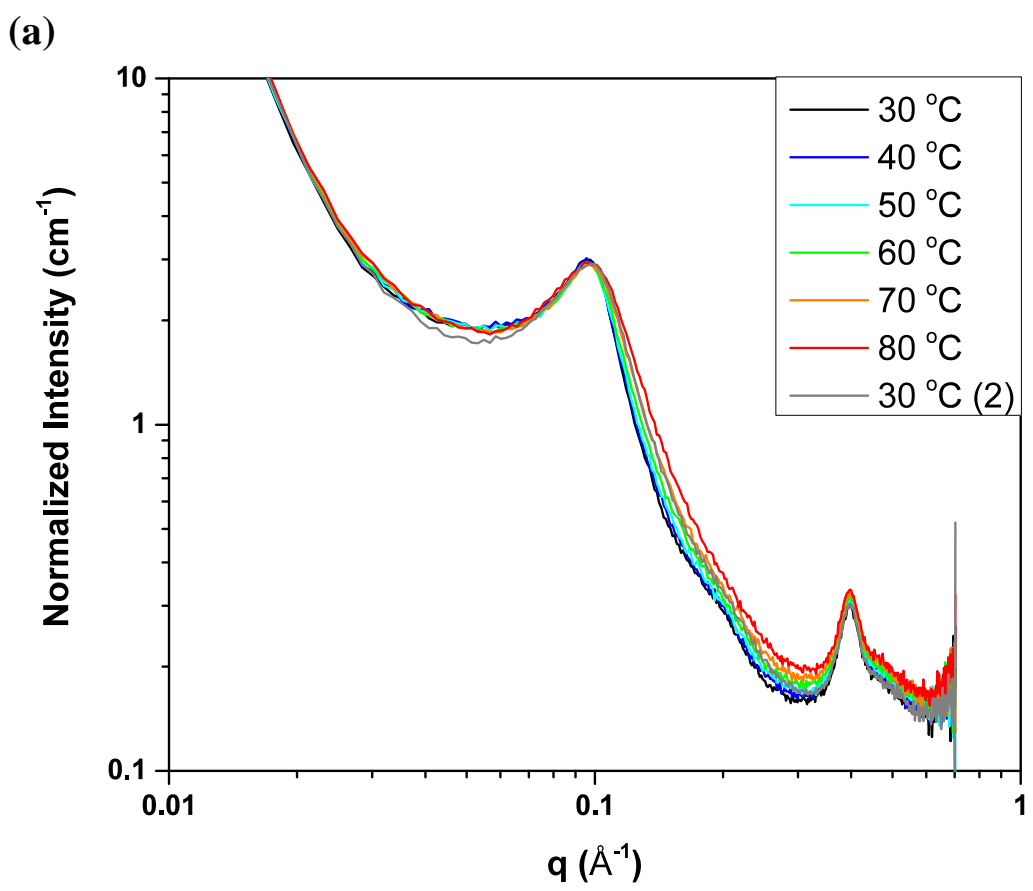

(b)

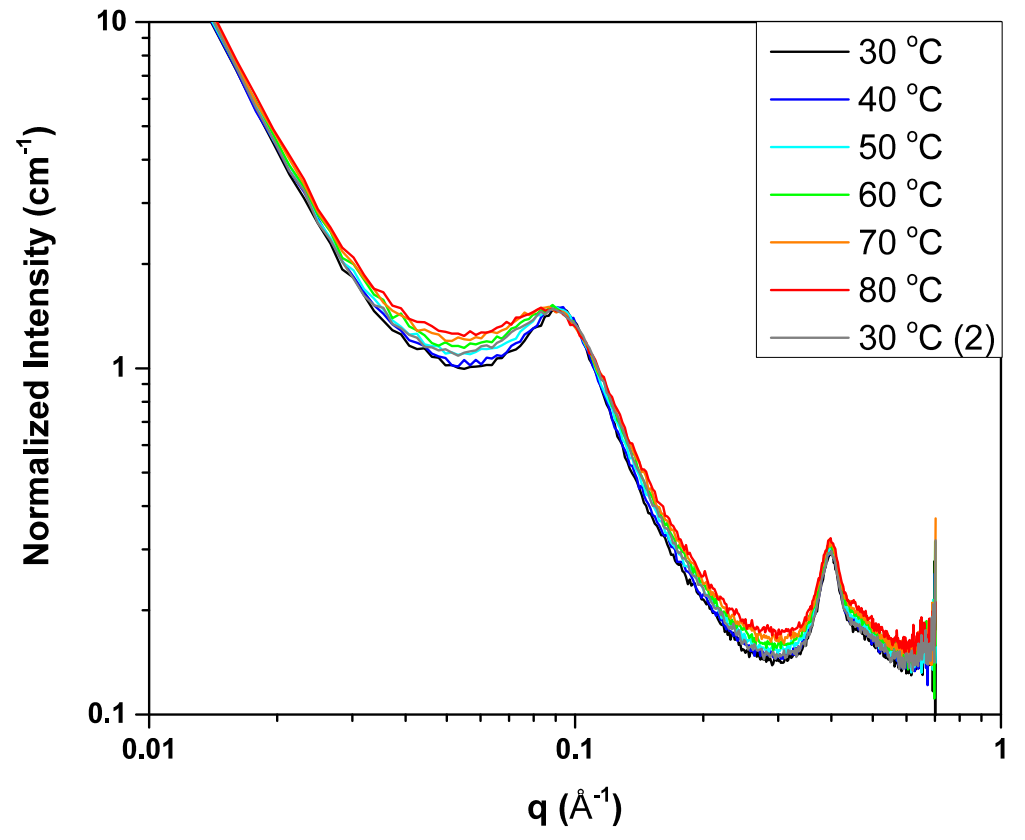




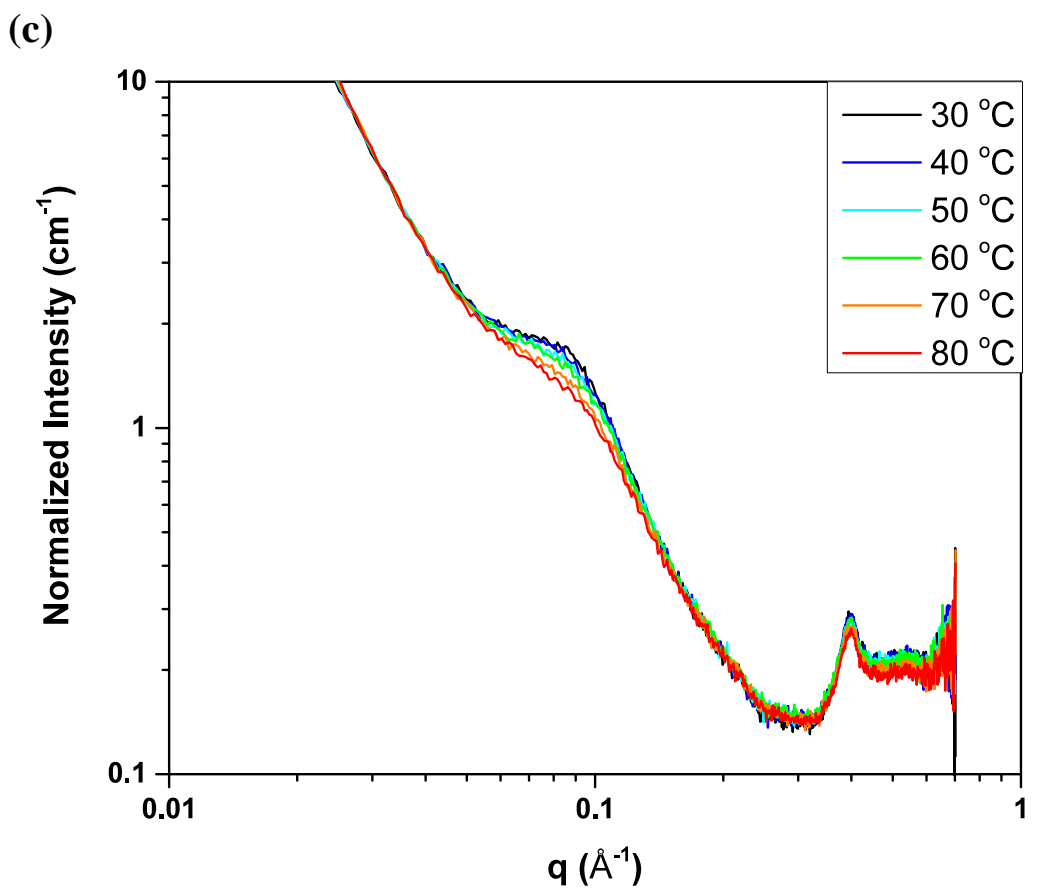

Figure S5. Variable temperature SAXS of (a) P(I-ran-SS)-7.2-TEA, (b) P(I-ran-SS)-7.2-TPA, and (c) $\mathrm{P}(\mathrm{I}-\mathrm{ran}$-SS $)-7.2-\mathrm{TEA}$ from $30^{\circ} \mathrm{C}-80^{\circ} \mathrm{C}$ in $10 \mathrm{~K}$ temperatures steps. 


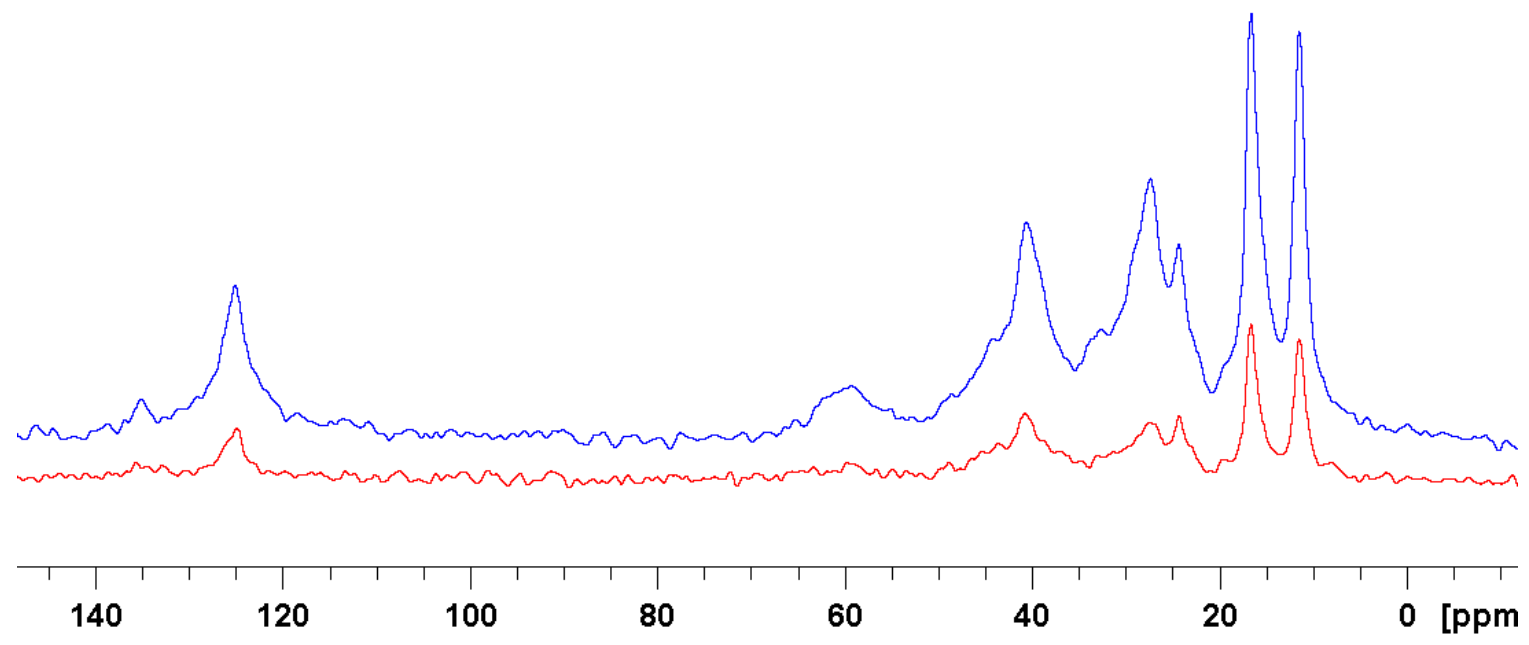

Figure S6. ${ }^{13} \mathrm{C}$ CP spectra of P(I-ran-SS)-7.2-TPA with spin lock times of $0.01 \mathrm{~ms}$ (blue) and 1 ms (red).

(a)

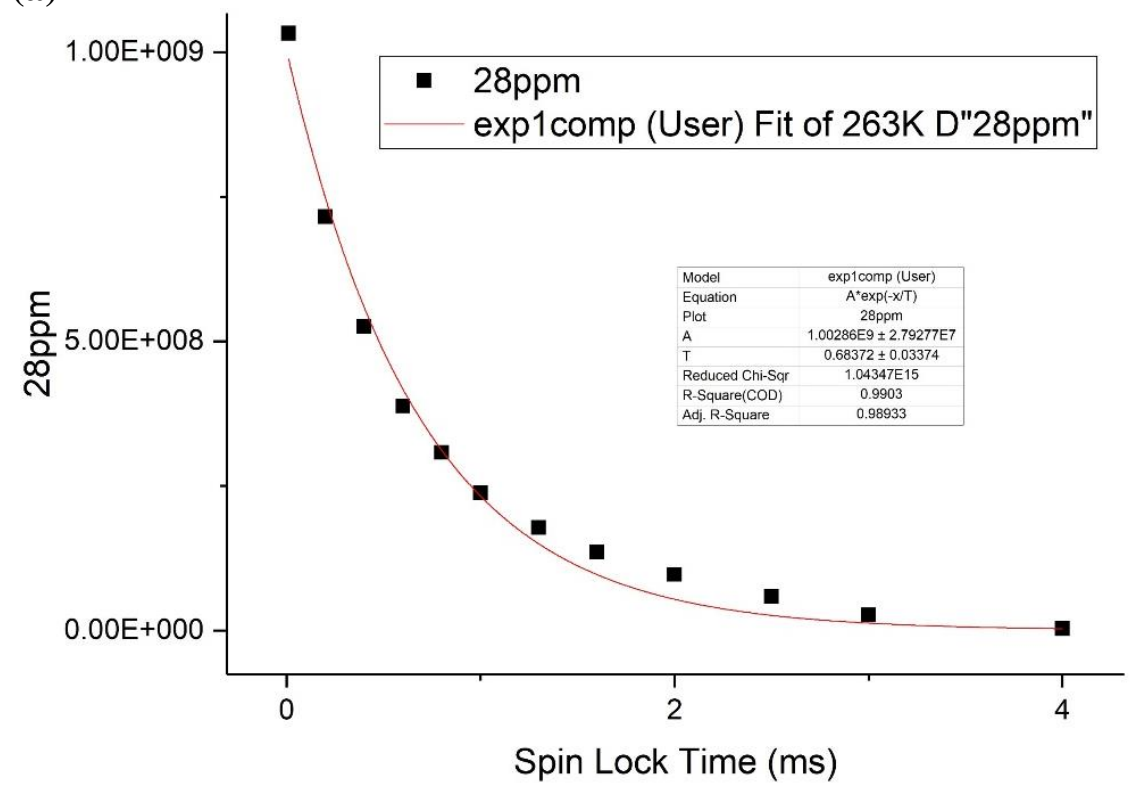


(b)

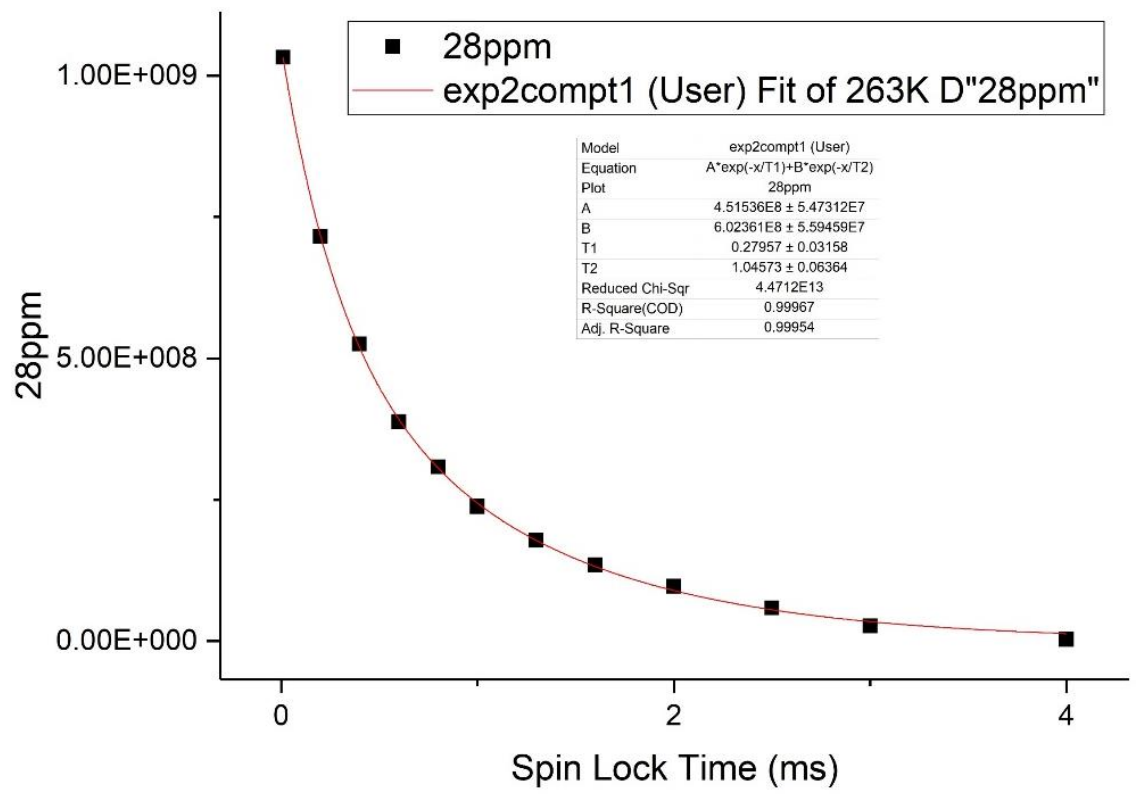

(c)

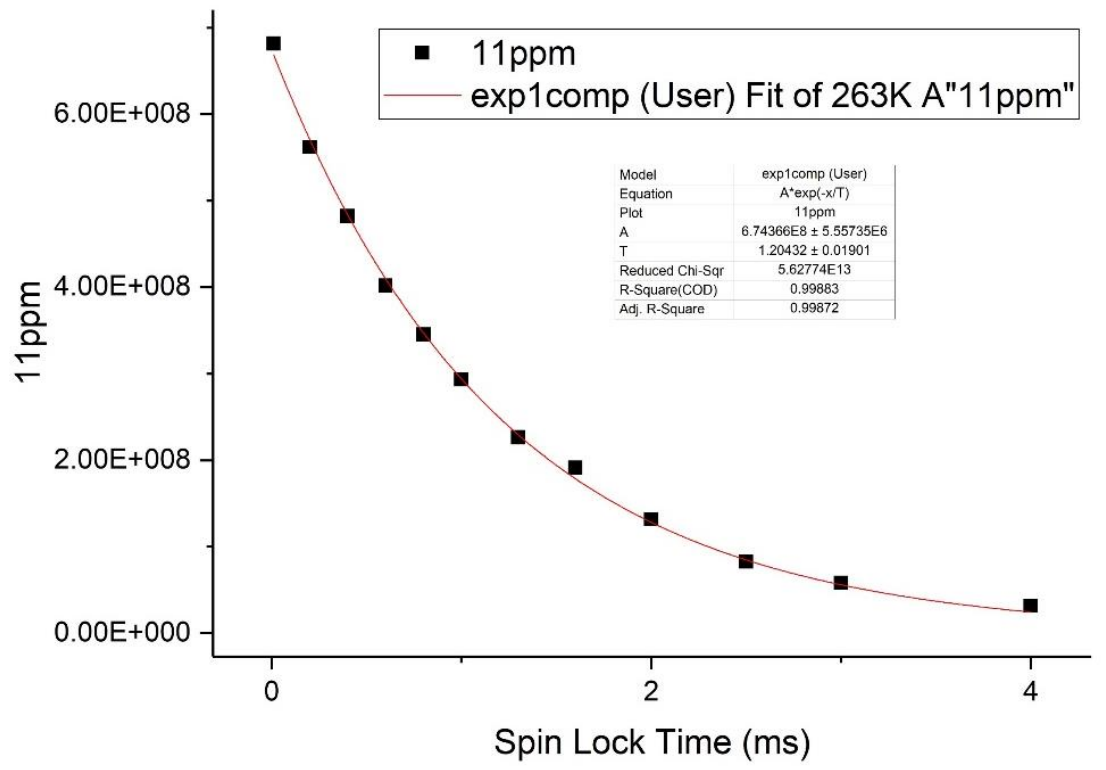

Figure S7. $\mathrm{T}_{1} \rho$ relaxation curve fits for P(I-ran-SS)-7.2-TPA at 263K. (a) Single-exponential fit of the isoprene signal at $28 \mathrm{ppm}$, which fails to accurately fit the experimental data. (b) $\mathrm{Bi}$ exponential fit of the isoprene signal at $28 \mathrm{ppm}$, which produces a much better fit. (c) Singleexponential fit of the counterion signal at $11 \mathrm{ppm}$, which provides an accurate fit of the experimental data. 
Table S4. Fitting Results for P(I-ran-SS)-7.2-TMA T ${ }_{1 \rho}$ Relaxation Experiments (contact time $=0.3 \mathrm{~ms}$ ). Superscripts $\mathrm{s}$ and I stand for short and long, respectively. The $\delta 17 \mathrm{ppm}, 28 \mathrm{ppm}$, and $41 \mathrm{ppm}$ peaks all correspond to the isoprene backbone. The consistency of the biexponential fitting results for all three peaks strongly support the existence of two isoprene dynamic components due to charge-induced aggregation. The fitting results of the $28 \mathrm{ppm}$ peak has been shown in Table 2 and are re-displayed here for comparison with results from other peaks.

\begin{tabular}{|c|c|c|c|c|c|c|}
\hline $\mathbf{T}(\mathbf{K})$ & $\begin{array}{c}\mathrm{T}_{1 \mathrm{\rho}}^{\mathrm{s}} \\
(\mathbf{1 7 p p m )})\end{array}$ & $\begin{array}{c}\mathrm{T}_{1 \rho}^{\mathrm{l}} \\
(17 \mathrm{ppm})\end{array}$ & $\begin{array}{c}\mathrm{T}_{1 \rho}{ }^{\mathrm{s}} \\
(28 p p m)\end{array}$ & $\begin{array}{c}T_{1 \rho}{ }^{l} \\
(28 p p m)\end{array}$ & $\begin{array}{c}T_{1 \rho}{ }^{s} \\
(41 p p m)\end{array}$ & $\begin{array}{c}T_{1 \rho}{ }^{I} \\
(41 p p m)\end{array}$ \\
\hline 263 & $0.98 \pm 0.15$ & $7.1 \pm 1.5$ & $0.64 \pm 0.09$ & $6.7 \pm 0.7$ & $0.66 \pm 0.08$ & $6.0 \pm 0.6$ \\
\hline 273 & $1.04 \pm 0.09$ & $6.2 \pm 0.5$ & $0.63 \pm 0.04$ & $6.5 \pm 0.3$ & $0.68 \pm 0.05$ & $6.0 \pm 0.3$ \\
\hline 283 & $1.08 \pm 0.02$ & $6.1 \pm 1.2$ & $0.71 \pm 0.04$ & $6.1 \pm 0.3$ & $0.66 \pm 0.08$ & $5.4 \pm 0.5$ \\
\hline
\end{tabular}

$\mathrm{T}_{1 \rho}$ relaxation measurements invoke cross polarization, which selectively emphasizes signals from rigid segments, so the amplitude of each $\mathrm{T}_{1 \rho}$ relaxation component does not reflect its real population. In all the two-component curve fits, the amplitude ratios of the two fractions are close to $1: 1$, while due to the poor $\mathrm{CP}$ efficiency of the isoprene matrix, its actual populations could be much larger than what the fitting results indicate. Poor CP efficiency could also be the reason the counterions dispersed in the matrix were not detected by $\mathrm{T}_{1 \rho}$ relaxation experiments.

The $\mathrm{T}_{1 \rho}$ of the N-methyl moiety in P(I-ran-SS)-7-TMA was measured in a separate series of experiments because sufficient signal strength of the $\mathrm{N}$-methyl group required longer contact time due to its low CP efficiency (likely as a result of the rotational freedom of the TMA cation). Therefore, $1.5 \mathrm{~ms}$ of contact time was used to track its $\mathrm{T}_{1 \rho}$ relaxation behavior. Similar to the TPA 
methyl signal of the P(I-ran-SS)-7.2-TPA sample, the relaxation curves of N-methyl in P(I-ranSS)-7.2-TMA at $\delta 55 \mathrm{ppm}$ are best fit by a single-exponential model for the entire temperature window studied (Table S3). To eliminate the possibility of the long contact time concealing a possible short- $\mathrm{T}_{1 \rho}$ component, $\mathrm{T}_{1 \rho}$ of the isoprene signal at $\delta 28 \mathrm{ppm}$ signal was calculated from the same experiment. The peak decay could only be well fitted by a bi-exponential model, thus confirming that the $\mathrm{T}_{1 \rho}$ of the $\mathrm{N}$-methyl group signal is indeed single-exponential. Additionally, the fitting results for the $\delta 28 \mathrm{ppm}$ peak differ slightly between the two contact times used, resulting from somewhat different selection of the population contributing to the signals.

Table S5. Fitting Results for P(I-ran-SS)-7.2-TMA T 1p $_{\rho}$ Relaxation Experiments (contact time $=1.5 \mathrm{~ms})$. Superscripts $\mathrm{s}$ and $\mathrm{I}$ stand for short and long, respectively. The $\delta 28 \mathrm{ppm}$ peak corresponds to the isoprene backbone, while the $\delta 55 \mathrm{ppm}$ peak results from the methyl group of the TMA counterion.

\begin{tabular}{cccc}
\hline $\mathbf{T}(\mathbf{K})$ & $\begin{array}{c}\mathbf{T}_{\mathbf{1}}{ }^{\mathbf{s}} \\
(\mathbf{2 8 p p m )}\end{array}$ & $\begin{array}{c}\mathbf{T}_{\mathbf{1}}{ }^{\mathbf{l}} \\
\mathbf{( 2 8 p p m )}\end{array}$ & $\begin{array}{c}\mathbf{T}_{\mathbf{1}} \\
\mathbf{( 5 5} \mathbf{p p m})\end{array}$ \\
\hline 263 & $0.8 \pm 0.2$ & $7.5 \pm 0.4$ & $6.1 \pm 0.4$ \\
273 & $1.2 \pm 0.3$ & $9.4 \pm 1.6$ & $6.2 \pm 0.3$ \\
283 & $1.3 \pm 0.3$ & $8.5 \pm 2.7$ & $4.9 \pm 0.4$ \\
\hline
\end{tabular}

Table S6. Fitting Results for the Polyisoprene $T_{1 \rho}$ Relaxation Experiment. 


\begin{tabular}{cc}
\hline $\mathbf{T}(\mathbf{K})$ & $\mathbf{T}_{1 \rho}(\mathbf{m s})$ \\
\hline 253 & $0.63 \pm 0.04$ \\
263 & $0.95 \pm 0.05$ \\
273 & $1.81 \pm 0.06$ \\
283 & $3.51 \pm 0.07$ \\
\hline
\end{tabular}

The different $T_{1 \rho}$ relaxation behaviors of the various moieties offers a possibility to conduct spin diffusion experiments with a gradient created by a $T_{1 \rho}$ filter. In Figure S8, at the onset of the spin diffusion (blue), the counterion peaks $(\delta 11,16$, and $60 \mathrm{ppm})$, which have a longer $\mathrm{T}_{1 \rho}$ relaxation time, are at their highest. The isoprene signals cannot be completely eliminated by the $\mathrm{T}_{1 \rho}$ filter as they have a long- $\mathrm{T}_{1 \rho}$ component, as discussed in the main text. The counterion signals decrease in intensity at increasing diffusion time, as their magnetization diffuse away to surrounding domains. In the meantime, the isoprene signals at $\delta 28$ and $\delta 41 \mathrm{ppm}$ gain strengths, by receiving magnetization from the counterions and the long- $\mathrm{T}_{1}$ isoprene units. The equilibrium is reached between 3 and $10 \mathrm{~ms}$. Using the spin diffusion coefficient of $0.4-0.8 \mathrm{~nm}^{2} / \mathrm{ms}$ for hydrocarbon polymers, ${ }^{4}$ estimate of separation between the counterion cluster and the matrix is likely in the order of $3-5 \mathrm{~nm}$. Note that the spin diffusion in this experiment involves the $\mathrm{z}$ component of the spins, the diffusion coefficient of which is a factor of approximately $3-4$ of that of the diffusion during spin lock, which occurs during the $\mathrm{T}_{1 \rho}$ relaxation experiments. 


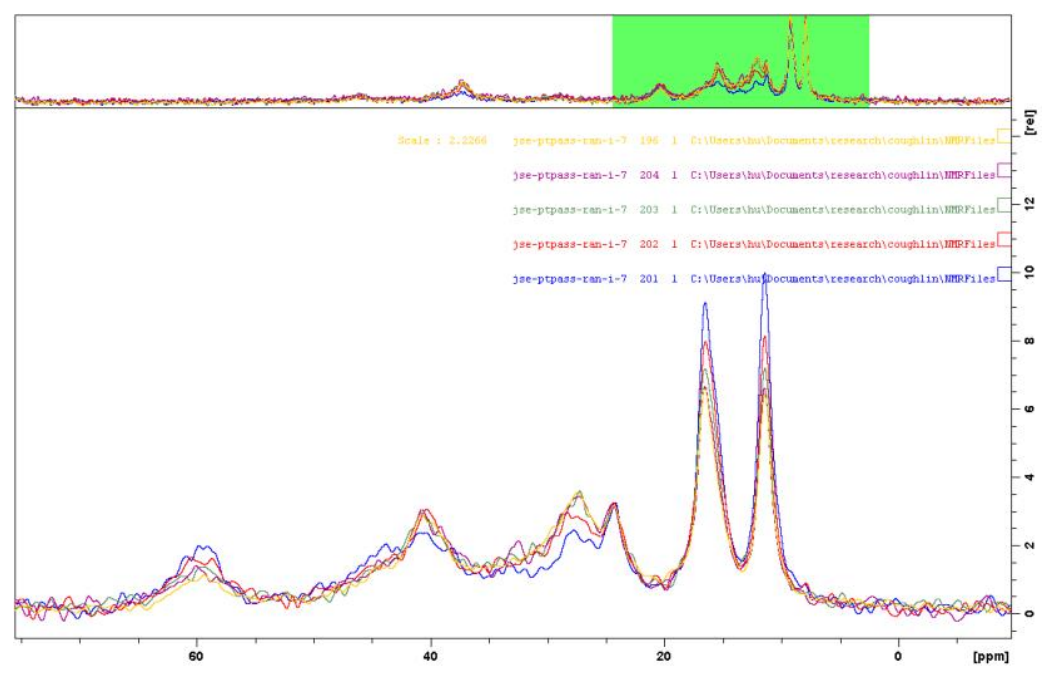

Figure S8. Spin diffusion experiments of P(I-ran-SS)-7.2-TPA with a $2 \mathrm{~ms}_{1 \rho}$ filter. Experiments were conducted at 263K. Diffusion time: 0.01 (blue), 1 (red), 3 (green), and 10 (purple) ms. The equilibrium spectrum (yellow) is plotted for reference.

\section{Thermal Analysis}

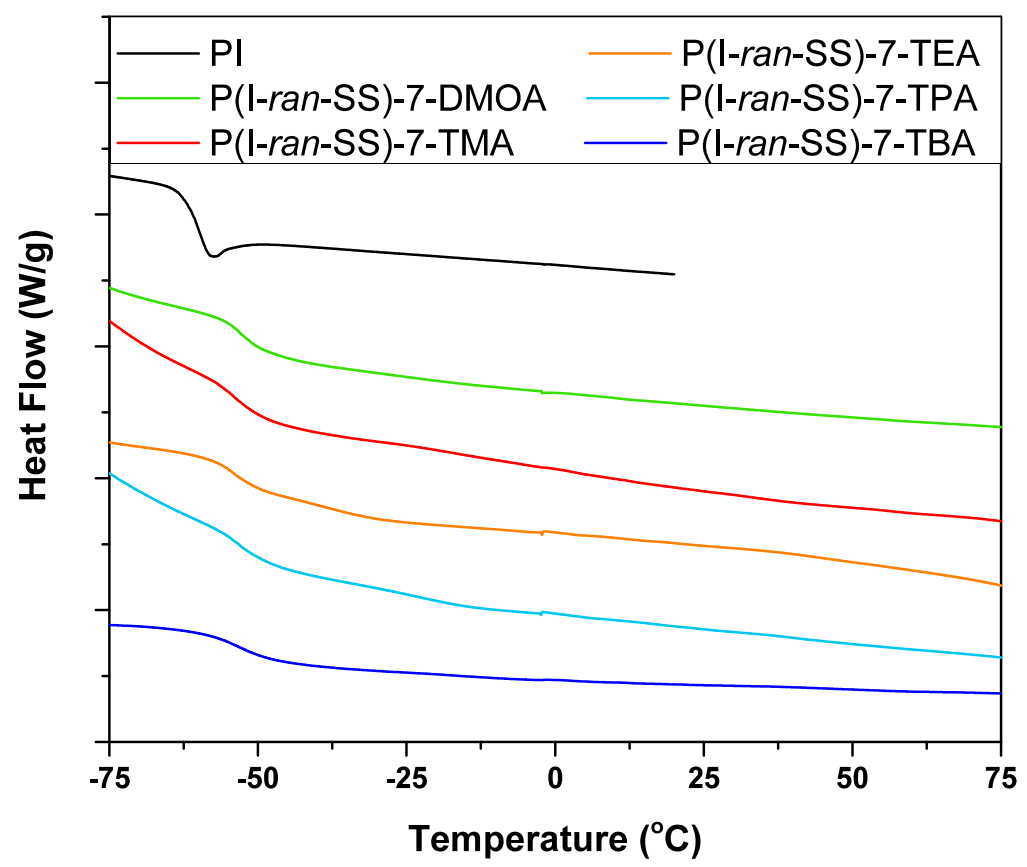

Figure S9. DSC thermograms for the PI homopolymer and P(I-ran-SS)-7.2-x copolymer series. 


\section{Rheology Analysis}

The SAOS data, G' and G", were merged into master curves and expressed in continuous relaxation time spectra shown point-wise in Figure $\mathrm{S} 10$. The $\mathrm{H}(\tau)$ data points were approximated using the proposed power law model (Equation 3 in the main paper)

$$
\mathrm{H}(\tau)=\mathrm{H}_{0}\left[1+\left(\tau / \tau_{\mathrm{g}}\right)^{-\mathrm{n}}\right] \text { for } \tau<\tau_{\max }
$$

and $H(\tau)=0$ above $\tau_{\max }$. The parameters of the continuous power law spectrum (model) were determined with IRIS Rheo-Hub (Table S7) where $\mathrm{H}_{0}$ is the spectrum value in the horizontal part of the spectrum, $\tau_{\mathrm{g}}$ is the transition time scale for the upturn, and $\mathrm{n}$ in the slope in the short-time region. The spectrum cut off at $\tau_{\max }$ is estimated, but could be larger. A better estimate for $\tau_{\max }$ would require SAOS data at much lower frequencies, which were not accessible. 

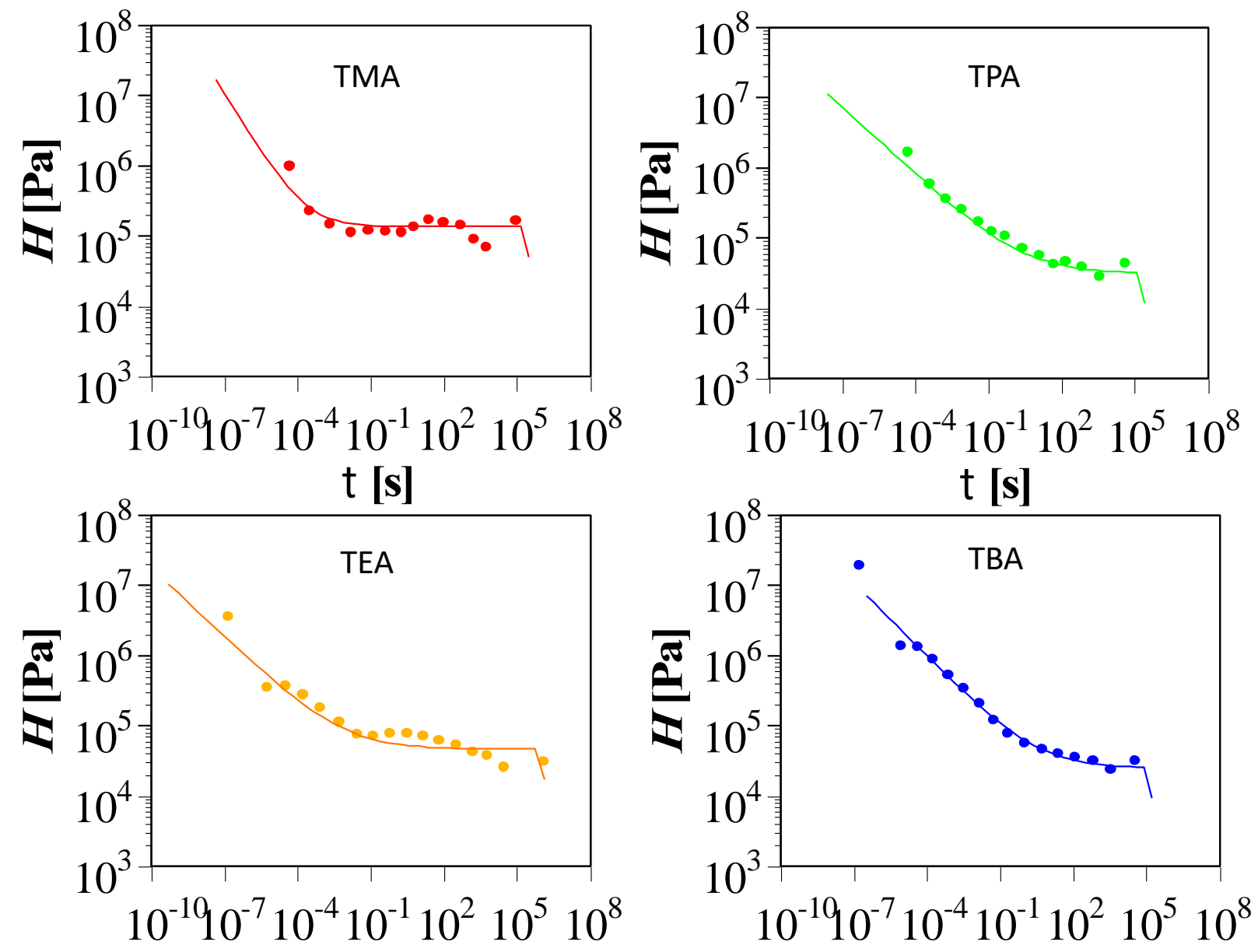

[s]

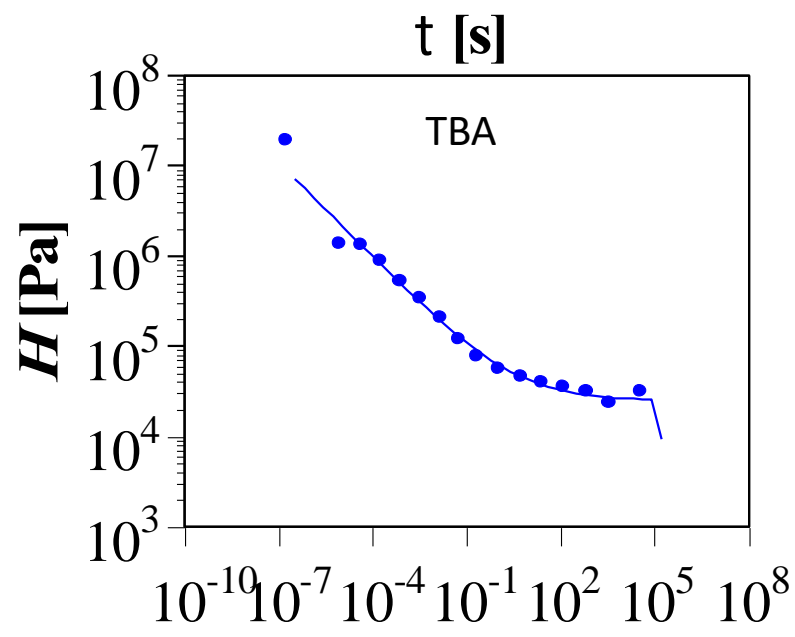

[s]

Figure S10. Data points belong to the continuous relaxation time spectrum of the four copolymers as determined from SAOS data using IRIS Rheo-Hub (method of Baumgäetel and Winter). ${ }^{5,6}$ The lines belong to the proposed model for $\mathrm{H}(\tau)$. 

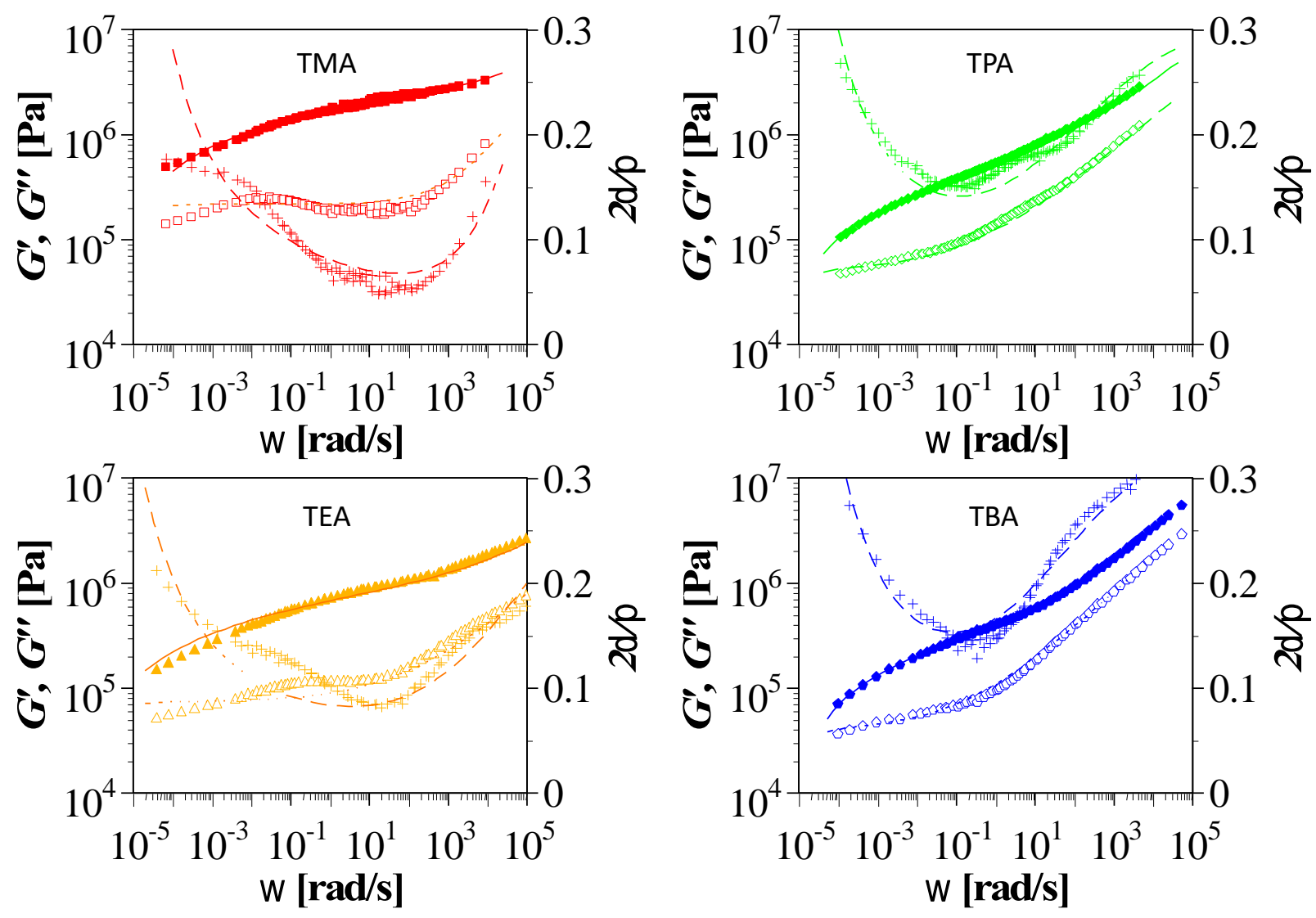

Figure S11. Representation of the measured SAOS data (data points) of the four copolymers using the proposed continuous relaxation time spectrum. The continuous line are calculated with Eq. 3 using the parameter values in Table S7 for the approximation of G', G', and the loss angles (scaled with $\pi / 2$ for 90 degrees).

Table S7: Parameters From the Continuous Power Law Spectrum Function.

\begin{tabular}{ccccc}
\hline Counterion & $\begin{array}{c}\text { Modulus Ho } \\
(\mathbf{P a})\end{array}$ & $\begin{array}{c}\tau_{\mathbf{g}} \\
(\mathbf{s})\end{array}$ & $\mathbf{n}$ & $\begin{array}{c}\tau_{\max } \\
(\mathbf{s})\end{array}$ \\
\hline TMA & $1.38 \times 10^{5}$ & $2.29 \times 10^{-4}$ & 0.56 & $2.82 \times 10^{5}$ \\
TEA & $4.67 \times 10^{4}$ & $6.77 \times 10^{-3}$ & 0.33 & $1.26 \times 10^{6}$ \\
TPA & $3.16 \times 10^{4}$ & 2.40 & 0.32 & $2.45 \times 10^{5}$ \\
TBA & $2.51 \times 10^{4}$ & 3.31 & 0.35 & $1.51 \times 10^{5}$ \\
\hline
\end{tabular}




\section{References}

(1) Kinning, D. J.; Thomas, E. L. Hard-Sphere Interactions between Spherical Domains in Diblock Copolymers. Macromolecules 1984, 17 (9), 1712-1718.

(2) Benetatos, N. M.; Chan, C. D.; Winey, K. I. Quantitative Morphology Study of CuNeutralized Poly(Styrene-Ran- Methacrylic Acid) Ionomers: STEM Imaging, X-Ray Scattering, and Real-Space Structural Modeling. Macromolecules 2007, 40 (4), 1081-1088.

(3) Zhou, N. C.; Chan, C. D.; Winey, K. I. Reconciling STEM and X-Ray Scattering Data To Determine the Nanoscale Ionic Aggregate Morphology in Sulfonated Polystyrene. Macromolecules 2008, 41 (16), 6134-6140.

(4) Chen, Q.; Schmidt-Rohr, K. Measurement of the local ${ }^{1} \mathrm{H}$ spin-diffusion coefficient in polymers. Solid State Nucl. Mag. 2006, 29, 142-152.

(5) Baumgärtel, M.; Winter, H. H. Determination of Discrete Relaxation and Retardation Time Spectra from Dynamic Mechanical Data. Rheol. Acta 1989, 28 (6), 511-519.

(6) Baumgärtel, M.; Winter, H. H. Interrelation between continuous and discrete relaxation time spectra. J. Non-Newtonian Fluid Mech. 1992, 44, 15-36. 